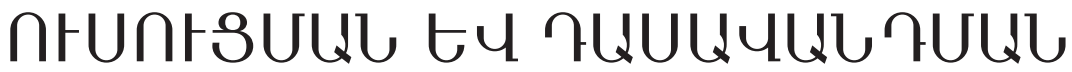 \\ UtคกาคนL
}

\begin{abstract}
SUZANNE SMITH
Doctor of Psychological Sciences Chief Clinical Officer for Autism Integrative Therapies, California, USA
\end{abstract}

SEDRAK SEDRAKYAN

Ph.D, Rector of University of Practical Psychology and Sociology "Urartu”, Armenia

\section{PARENT TRAINING FOR CHILDREN WITH AUTISM}

From a behavioral perspective, culture is the full range of learned human behavior patterns (Samadi\&McConkey, 2011). Considering this definition, ABA in itself has its own culture (Miller, 2006). However, minimal research has been conducted specifically on the role of immigrant families and providing culturally sensitive ABA based parent training. Behavior analytic principles and interventions originated in the United States, and seem more efficiently applied to the mainstream cultural values in the United States (Samadi\&McConkey, 2011). It is notable that much of the research conducted in ABA focuses on individuals who were born or have been well assimilated into what may be considered a common set of cultural values subscribed to in the United States. Although parents in the United States need training to apply the principles of $\mathrm{ABA}$ in managing their children's behavior, it is approached in a more overarching context of social norms common to the United States. However, practitioners applying behavior analysis using skill sets they have developed in a more mainstream perspective (ABA used in the United States) find it difficult to generalize the use of these skill sets to families in many immigrant communities. Therefore, it is important that the field of Applied Behavior Analysis develop approaches with a focus on the individual characteristics of families, especially those of immigrant populations. The specific interventions used must align with what is accepted of the culture in the home. This is an urgent issue, since the number of children being diagnosed with autism spectrum disorders (ASD) is dramatically rising in immigrant communities.

This study examines ABA based individualized parent training as an important agent for decreasing the target behaviors of children diagnosed with autism spectrum disorders. This study is conducted in the homes of immigrant families who traditionally do not use positive reinforcement as a primary way to change behavior.It supports the notion that there is a functional relationship between ABA based parent intervention and reduction of symptoms using ABA strategies.

This study focuses on how parent disciplinary tactics contribute to the positive or negative behaviors of children with Autism Spectrum Disorders (ASD). Research has shown that Applied Behavior Analysis (ABA) is an extremely effective treatment for children with ASD, and that positive reinforcement is more successful than punitive techniques when the goal is 
cessation of undesirable behaviors (Piazza, 2005). This research paper concentrates on the immigrant community in particular. The reason for this is that research on ASD in general, as well as research on the effectiveness of ABA in the treatment of these disorders, is limited in this population.

In many immigrant communities, the norm for parents is to display punitive (aversive) disciplinary techniques, resulting in little success at reducing problem behaviors long term for children with ASD (Samadi\&McConkey, 2011). Ultimately the hypothesis is that training parents on how to appropriately use positive reinforcement to differentially reinforce desired behaviors over problem behaviors will result in a longterm reduction of the undesired behaviors. The steady growth in numbers of treatment plans developed by ABA practitioners which show positive outcomes achieved in the lives of the treated individuals and their families speaks to the success of treatment strategies based on the principles of ABA as applied to non-immigrant families. There is also a significant body of research in peer-reviewed journals (e.g., Journal of Applied Behavior Analysis) related to the practice of ABA, supporting and legitimizing the conceptual basis on which treatment through ABA is founded.

\section{Method}

The purpose of this study is to demonstrate, through case studies, the effectiveness of parent training on the use of non-aversive behavior management techniques, which may run somewhat counter to the parent's pre-existing beliefs and practices on this matter. To demonstrate success in using parent-training techniques, we selected three immigrant parent-child dyads to assess and subsequently treat in the context of the family eating dinner. It was important to conduct research in a more common and authentic situation such as this, to determine how effective these strategies would be in addressing some of the more seemingly extreme behavior problems known to be exhibited much more often by children diagnosed with ASD than by more typically developed individuals. These extreme actions include behaving inappropriately (i.e., removing clothing), acting aggressively (i.e., attempting to bite parents), and being disruptive (i.e., throwing food).

\section{Participant Selection}

The parents were referred to participate in this study from the Glendale Community Center and from the Autism Outreach program. The dyads selected met the following criteria: (1) the parent had a child diagnosed with autism; (2) the child demonstrated one or multiple problems at home; (3) the child was between 2 to 17 years old; (4) the parent was willing to participate in parent training and implement the learned skills with his or her child diagnosed with autism; (5) parents were from different ethnic groups, and they had been residing in the U.S. for fewer than 10 years; finally, (6) parents had had little to zero training in the implementation of ABA based behavior management techniques.

\section{Setting}

Although problem behaviors exhibited by the children often occurred in many other settings, the parents agreed that meals were occasions where problem behaviors were most likely to occur and could predictably be viewed in this setting. The simulated atmosphere allowed parents to implement and test various ABA techniques within the natural routine. For example, during the mealtime simulation, parents and children sat at the table as if it was a usual supper, with a meal on the table. Parent training interventions and observations took place in this setting.

Participants

Chris and his mother Gina. Chris, a 5-year-old boy, was energetic and demonstrated an obsession with toy cars. He was noncompliant and the parent was 
concerned that he did not regularly follow directions. He engaged in gazing about during tasks, and did not respond when called by name. He often became rigid about routines and would resist change in his schedule. He had a history of normal development up through the time that he began school. At this point, his parents began to notice a regression of skills, and enrolled him in speech therapy. A family physician referred Chris to Autism Treatment Center, where he was diagnosed with Autism Spectrum Disorder. Chris was living with his biological parents in an upscale neighborhood. Chris was living at home with both biological parents. His mother was the primary caregiver and was a teacher; however, she was not currently working. Chris's father was often out of town on business during the work week. Chris's mother Gina was 35 years old. She moved to the United States from Poland. As she mentioned during the interview, she kept the traditional family rules in the raising of her son, Chris.

Avo and his mother Martha. Avo, a 6-year-old boy, demonstrated delayed development with language; however, motor milestones were within normal limits. Avo was diagnosed with autism by a psychologist at the regional center. Avo spoke and understood both English and Spanish; however, his primary language was English.He was able to speak in short sentences, but had difficulty communicating his ideas. Often his sentences were jumbled, and only the beginning or ending of the sentences was intelligible. When he got excited his speech became less intelligible. Avo's mother Martha was 34 years old. She immigrated to the United States 4 years ago from Ecuador. She was Avo's primary caregiver and did not work. Avo's father was a construction worker. During the interview, the father expressed that he was concerned about raising the child in a different manner than he was familiar with, based on his cultural values.

Abby and her mother Rebecca. 7-yearold Abby was diagnosed with autism at the age of 3. Rebecca reports that Abby used about 20 words until she was 2, then lost all her words. Rebecca stated that, since Abby had lost her words, her noncompliance had become more frequent, occurring throughout the day for "any reason". Abby speaks, reads, and writes English. She understands some Farsi (e.g., basic directions such as "come here", "give me _", etc.). Rebecca and her husband arrived in the U.S. from Iran about 9 years ago. They currently reside in a singlefamily home in the San Fernando Valley. Rebecca's husband is a physician and she is not working.

\section{Materials}

Baseline, the treatment of Parent Training and data recorded for the treatment phase data took place in simulated conditions at home, imitating the usual daily routine at the dinner table. Materials included those that would aid in most closely simulating a natural environment (plates, utensils, cups, etc.) where the parent training was taking place. The location of everything in the house, including where the dining table and chairs were situated, remained the same. Items preferred by the children continued to be used in this setting as well (a preferred cup, plate, favorite toy often brought to the diner table by the child, etc.).

\section{Measures}

All targeted problem behaviors exhibited by the children were measured using a total count of each occurrence during a one-hour session. When a targeted problem behavior occurred, the recorder also recorded a plus or minus, with plus signifying that the parent responded to the targeted behavior using a correct treatment technique and minus signifying that the parent used a non-treatment aversive response to the child's behavior. The percent of correct responses to the behaviors out of the total 
opportunities per session (occurrence of targeted behavior) were calculated.

\section{Dependent Variable}

There were two dependent variables that were being measured in this study: the total number of targeted problem behaviors per session and the percent of these targeted behaviors that were responded to as specified by the treatment plan. The form of positive reinforcement that was programmed in the treatment plans for the parent to use was simply specific verbal praise upon the occurrence of replacement behaviors which included any gestural or spoken attempt to get the parent's attention and/or using a utensil and/or napkin when eating. These replacement behaviors were programmed into each of the children's treatment plans. The targeted behaviors for increase were the same for each child. If a parent reinforced any occurrences of these targeted behaviors for increase, a mark was recorded for the occurrence of the behavior as well as a plus for the parent's correct implementation of the reinforcement procedure. If the parent gave no response following the occurrence of a targeted behavior for increase, the occurrence of a targeted behavior was recorded, as well as a minus for incorrect implementation of the treatment procedure. Thus, not only was the child's behavior measured as a dependent variable, but the parent's programmatically correct responses were also measured as a dependent variable. In this way we arrive at a total percentage indicating to what degree a parent was successfully using positive reinforcement.

Conversely there were also two dependent variables measured for decrease. Data was recorded on the total occurrences of behaviors targeted for decrease and, similar to the approach toward measurement of positive reinforcement above, data was recorded reflecting what percent of targeted behaviors for decrease were responded to by the parents using punitive (aversive) techniques.

This study focused more generally on behaviors that were disruptive in nature. None of these individuals displayed selfinjurious behaviors or significant aggression towards their parent other than some mild forms of pushing the parent away. Forms of behavior counted as within the study's broader definition of "disruptive" varied between each child. Chris pushed items of the table such as his plate, cup, utensils, etc.; Avo yelled loudly and repeatedly while bouncing in his chair, banged his fists on the table, and at times simultaneously yelled loudly; Abby behaved very similarly to Avo with loud repetitive yelling, bouncing in her chair and banging her fists on the table. These are the definitions for the behaviors measured for decrease for each of the children participating with their parents in this study, and these definitions are what are listed on the data recording form.

Independent Variable

The independent variable/ treatment used in this study consisted of parent training. The parent training treatment consisted of parent-child dyads receiving one to one parent training by a BCBA within the home setting. The behavior analyst conducting parent training described the treatment strategies, how they were arrived at through assessment, and gave examples of successful outcomes for other families with similar children engaging in similarly disruptive behaviors (though mentioning that each family presented their own circumstances and the success of these procedures could not be guaranteed in every situation). Parent training was conducted for all participants, and also included behavioral skills training, modeling, and feedback. Parents were trained on the implementation of differential reinforcement using positive reinforcement to increase desired behavior and, in the case of all these particular participants, planned ignoring (extinction 
of behaviors maintained by attention) to decrease problem behavior.

\section{Design}

In this study a multiple baseline design was used across the three parent-child dyads, which included Gina and Chris, Martha and Avo, and Rebecca and Abby, respectively. A multiple baseline design seemed most appropriate as we were studying the effects of a treatment that could not be withdrawn as in a withdrawal/ reversal type design. The parents, having been taught to correctly implement ABA based treatment procedures, can only continue to produce data post treatment as it would not seem reasonable to ask parents to stop implementing the procedures correctly once they had learned to do so. Baseline data was recorded for the first dyad and then the treatment implemented after three recording sessions while the other two children remained in baseline. The treatment for the second dyad was implemented two sessions after the first in order to build evidence toward the treatment being the variable correlated with the change in behavior and not some other change in the children's environments. Finally, after another two sessions following the second dyad, the third dyad began the treatment phase. The extension of these baselines provided evidence that the only variable in each of these children's environments that can be correlated with behavior change is the implementation of the treatment in the form of parent training.

\section{Baseline}

Recording of baseline data for each dyad began on the same date in their separate residences. Baseline conditions were simply the typical evening meal conditions present in the families' regular routines. The parents had limited if any training in the implementation of ABA strategies and interacted with their children and responded to their problem behaviors as is typical of any given evening meal in their home. The number of targeted behaviors that occurred over a one-hour period was recorded as well as the number of occurrences of these problem behaviors where the parent responded using aversive techniques. Thus, baseline data was recorded on both how many applications of aversive techniques were used by parents, as well as how many opportunities for the parent to use these techniques were provided by the children (frequency count of targeted problem behaviors exhibited by the children).

\section{Intervention.}

The treatment of Parent Training was taught in the simulated setting described above and then treatment data was recorded during the typical mealtime and associated environmental contexts as above with baseline. The only difference distinguishing the treatment phase from the baseline phase was that the parents had been trained on some basic behavioral techniques to use in response to problem behaviors from their children in place of the aversive responses used more prevalently in the baseline phase.

The parent training implementation had 2 distinct stages. In stage one, a behavior intervention plan was designed, based on the function of the child's target behaviors. In stage two, parent training sessions were conducted in order to teach parents the concepts of ABA, explaining the effectiveness of these strategies and how to use them without conflicting with their cultural concepts of child rearing. In this stage particularly, the main barriers to using positive reinforcement were emphasized. These barriers, imposed by the two parents' cultural beliefs, could be summed up in the following way: instead of rewarding their children's positive behaviors, parents in certain cultures feel that they have to demand, reprimand and intimidate children in order to keep them under control and compliance.

The cultural concepts were examined, 
revealing the necessity of replacing them with ABA approaches that were rejected, or in many instances used in an inappropriate manner, due to the incompatibility with their cultural beliefs, parenting skills, and family values.

The parent trainers, Board Certified Behavior Analysts (BCBAs), demonstrated the use of effective ABA strategies with the children during the simulated dinnertime routines. One BCBA worked with each dyad to complete the training in their home environment. After the demonstration, the parents were asked to practice the use of effective strategies with the children during the session. The behavior analyst gave the parent immediate feedback to confirm the correct usage of ABA strategies such as positive reinforcement. When the parent did not use the appropriate treatment plan procedure in responding to a problem behavior the BCBA gave the parent feedback about what the correct treatment procedure would have been for the incident of problem behavior and included on the spot training and demonstration of the procedure for the parent. The duration of parent training was one hour for each dyad.

Modeling Specific Effective Practices for Parents

The behavior analyst first modeled with the child and for the parent prior to the parent implementing the strategies. Behavior modeling allowed the parents to see the demonstration of the strategies and to understand how to implement these as listed on the Parent Checklist. Additionally, the behavior analyst reported that parents actively asked questions while referring to the Parent Checklist for clarification during the modeling portion of the training.

Guided Practice in Home Setting

Each parent directly engaged with his or her child in a simulated setting to practice

ABA techniques, both modeled by the behavior analyst and listed on the parent checklist. During the simulation, the behavior therapist was present and provided verbal and physical prompts, as needed, to guide parents' implementation of ABA techniques. For example, if the child engaged in appropriate behavior and the parent did not present positive reinforcement, the interventionist pointed to the reinforcement to prompt the parent. In another example, if the child engaged in non-compliance and was throwing food, and the parent did not address the child's problem behavior, the interventionist verbally prompted the parent to remove the preferred item and have the child pick up the food that was thrown on the floor.

Interobserver Agreement (IOA)

IOA data was recorded once during the baseline phase and once during the treatment phase for each parent. A checklist was implemented for recording parents' use of positive reinforcement. The primary and secondary observers recorded the occurrence and non-occurrence of parents' responses. The training sessions were video recorded. At the end of each observation period, the total number of occurrences and non-occurrences of parent behavior and proper implementation of ABA techniques, namely reinforcement, were recorded. A correct response was scored if the parent used positive reinforcement and an incorrect response was scored if the parents used a punitive consequence.

The IOA was calculated by dividing agreements by the sum of agreements plus disagreements with the resulting value multiplied by 100 to obtain a percent (\%) value (Cooper \& Heron, 2004).

Data for IOA was also recorded and calculated for the children's behavior. IOA data was recorded once during the baseline phase and once during the treatment phase for each parent. This was completed using a different method for calculating IOA than for the parent's behavior. The data for the targeted behaviors exhibited by the children were recorded as frequency per 
session. This involved a smaller number divided by a larger number and then multiplied by 100 to get a value for percent agreement between both recorders.

The results of IOA data for the parent training showed IOA that the data recorders agreed on what they recorded (between 85 and 90 percent). The IOA data for the recorders of the children's problem behaviors as well as replacement behaviors shows agreement of 95 to 100 percent. These IOA results are well within the range of indicating that the data recorded on the behavior of both the parents and the children is useful for interpretation and display of socially significant results.

Fidelity

In order to ensure the fidelity of implementation of intervention procedures, the primaryobserver recorded data on the interventionist's behaviors for all training sessions on the use of specific responses implemented by the behavior analyst with the parent during training sessions. These responses included: (1) behavior analyst demonstrating routine with child, (2) interventionist prompting and confirming parent behavior with child, (3) parent demonstrations of the procedures identified in the parent behavior checklist while the behavior analyst provided parent with critical feedback necessary to maintain acquired skills or practice newly learned skills, (4) behavior analyst allowing parent to demonstrate working hands-on with child during the training routine, (5) training setting being different from targeted natural environment, and (6) behavior analyst being present to provide any assistance or guidance to the parent in case of occurrence of problem behavior.

Data measuring the correct implementation of treatment plan procedures by the parents after the initial training corresponds with the data collected of percent of opportunities that the parent responded correctly by implementing treatment procedures as specified in the treatment plan. In this study the data concerning the fidelity of parent implementation is already addressed simply through the measurement of one of the two dependent behaviors being measured. Please see raw data for the percent of correct implementation of treatment procedures following the implementation parent training which is the treatment related to the behavior of the parents.

\section{Results}

Results from the study indicate that each child's behavior could be improved using treatments that rely strongly on positive reinforcement as a key component. This study has produced evidence that parents of differing cultures, who may find it difficult to incorporate the relatively high amount of positive reinforcement into their daily approaches to managing child behavior, can learn to implement evidence-based strategies with their children as a means of changing undesired behavior.

In dyad 1, during baseline, parents rarely used positive reinforcement, ranging between 0 and $5 \%$, with a mean of $3 \%$ per opportunities per session. However, after intervention was implemented, the data show an increased trend to a range of 60 $100 \%$, with a mean of $74 \%$. During baseline, Chris's undesirable disruptive behaviors, which include pushing a variety of items related to the mealtime environment off the table, occurred at a rate of 14-17 per hour, with a mean of 15.5, while the child's desirable/replacement behaviors of raising his hand for attention and eating his food correctly occurred at a rate of $0-5$ per hour, with a mean of 3. After intervention was implemented, the child's undesirable behaviors decreased to a range of between 5 and 7 occurrences with a mean of 3 per hour, and the desirable behaviors increased to a range of between 8 and 9 per hour, with a mean of 8.5 .

During baseline for dyad 2, parents rarely 
applied treatments from the treatment plan including reinforcement of replacement behaviors with a range of between 0 and 5 percent and a mean of 3 percent. Avo's problem behaviors, which included loud repetitive yelling, bouncing in his seat and banging his fist on the table, occurred between 17 and 22 times per hour, with a mean of 19 . Replacement behaviors occurred at a range of between 0 and 1 per hour, with a mean of .5 per hour. Avo's replacement behaviors included raising his hand for attention and eating appropriately with utensils. After parent training on treatment through positive reinforcement, parental use of treatment procedures rose to a range of between 80 and 100 percent and a mean of 90 percent. The problem behaviors decreased to $0-1$ per hour, with a mean of .5 per hour. Replacement behaviors increased to 19-22 per hour, with a mean of 20

During baseline for dyad 3, Abby's parent engaged in correct implementation of the treatment plan and its reinforcement procedures at a range of between 0 and 5 percent with a mean of 3 percent. Following parent training Abby's parent engaged in correct implementation of treatment procedures at a range of between 80 and 100 percent with a mean of 90 percent. Abby engaged in problem behaviorsat a rate of 16-21 per hour, with a mean of 20 . She engaged in the appropriate replacement behaviors of raising her hand for attention and eating appropriately with her utensils 15 to 20 occurrences per hour, with a mean of 17 per hour.

As for parent behavior, all three dyads performed similarly to one another. During baseline all dyads were providing a very low range, or no positive reinforcement at all. After intervention of parent training, parents all reached criteria of $100 \%$ use of positive reinforcement for alternative appropriate behavior.

IOA results ranged from 95 to $100 \%$ across dyads. The final intervention was calculated to be $100 \%$.

\section{Discussion}

This study investigated the effectiveness of parent training for enabling parents to

implement a function-based behavior intervention using positive reinforcement during the meal-time routine associated with high rates of child problem behavior in a home-based setting. Evaluating the effect of the parent training on ABA reinforcement techniques by parents was the key variable of interest in the study. Because of using reinforcement techniques, the children's problem behavior decreased, and the compliance increased.

Much of the peer reviewed literature in the field of behavior analysis related to the study of problem behavior exhibited by children with ASD does not include in depth assessment of variables related to fluency of caretakers (particularly parents) at implementing treatment plan procedures. Most of the literature has been limited by two aspects: (1) changes in child behavior being focused on as the only behavior change that needs to be measured without considering the fundamental role played by the behavior of parents in ultimately changing the behavior in children, and (2) treatment of child behavior being focused on skill deficits in the child only and not factoring the training and skill level of parents as a key environmental variable related to child problem behavior. This study was conducted by focusing on both the measurement of parent behavior and on the evaluation of the effect of parent training for reducing child problem behavior.

The objective of this research study was to determine if problem behavior displayed by a child with autism would decrease in the natural environment as a result of ABA based parent training. This study contributes to training parents in $\mathrm{ABA}$, and making it culturally acceptable and 
compatible through a deep understanding of applications of ABA, not conflicting with their cultural values and beliefs. Parents reported that they will use the ABA modalities for reducing children's target behaviors.

Nevertheless, the existing parent training programs do not address the cultural aspects of the training, which create resistance and conceptual differences from the parents' point of view for proper implementation of the ABA approaches (Koegel, 2005). The resistance, due to lack of cultural sensitivity, often prevents or distorts the correct and effective implementation of ABA. According to traditional parenting approaches in many cultures, addressing children's challenging behavior clearly favors only a punitive approach (Moes\&Frea, 2000). Parent perspectives of autism considerably vary, comparing them to a mainstream approach (Ali \&McConkey, 2011).

Possible limitations of this study pertain to maintenance and generalization of this intervention. As the cultural traditions do not include positive reinforcement as a primary way to control behavior, it is unknown whether the intervention will sustain.

Results from this study suggest that other cultures subscribing to punitive practices to primarily control children's behavior can benefit from ABA techniques. Positive reinforcement was shown to make a greater decrease in undesired behavior and a greater increase in desired behavior, when compared to a punitive approach.

Future research needs to consider the maintenance and generalizability of parent training for culturally diverse populations. Training may need to include general approaches that can be modified based on individual needs of the family.

To conclude, this study demonstrated the effectiveness of parent training on ABA related principles, namely reinforcement, on changing their children's behavior. Parents were able to decrease problem behavior and increase appropriate replacement behaviors by using positive reinforcement. Outcomes of this study showed distinct and obvious improvements in the behavior of the participating children particularly in the case of families where the use of positive reinforcement was very rare in the parent's baseline approach to the management of problem behaviors in the home. It can be stated that ABA techniques are effective with a wide variety of populations, even those that have not regularly used them in the past. 


\section{REFERENCES}

1. American Psychiatric Association. (2000). Diagnostic and statistical manual of mental disorders (4th ed., text rev.). Washington, DC.

2. Au-Yeung, S.K., Benson, V., Castelhano, M., \& Rayner, K. (2010).Eye movement sequences during simple versus complex information processing of scenes in autism spectrum disorder. Autism Research and Treatment, 2001 (Article ID 657383), 1-7.

3. Baker-Ericzén, M. J., Brookman-Frazee, L., \&Stahmer, A. (2005). Stress levels and adaptability in parents of toddlers with and without autism spectrum disorders. Research and Practice for Persons with Severe Disabilities, 30(4), 194-204.

4. Bristol, M.M., \&Schopler, E. (1983). Stress and coping in families of autistic adolescents. In M.E. Schopler\& G.B. Mesibov (Eds.), Autism in Adolescents and Adults (251-278). New York: Plenum Press.

5. Eikeseth, S. (2001). Recent critiques of the UCLA young autism project. Behavioral Interventions, 16(4), 249-264.

6. Koegel, R. L., Bimbela, A., \&Schreibman, L. (1996). Collateral effects of parent training on family interactions. Journal of Autism and Developmental Disorders, 26(3), 347-359.

7. Koegel, R. L., Vernon, T. W., \&Koegel, L. K. (2009). Improving social initiations in young children with autism using reinforcers with embedded social interactions. Journal of Autism and Developmental Disorders, 39(9), 1240-1251.

8. Lafasakis, M., \&Sturmey, P. (2007). Training parent implementation of discrete-trial teaching: effects on generalization of parent teaching and child correct responding. Journal ofApplied Behavior Analysis, 40(4), 685-689.

9. Moes\&Frea, D. R. (2000). Using family context to inform intervention planning for the treatment of a child with autism. Journal of Positive Behavior Interventions, 2(1), 40-46.

10. Murray, M.M., Ackerman-Spain, K., Williams, E.U., \&Ryley, A.T. (2011).Knowledge is power: empowering the autism community through parent-professional training. The School Community Journal, 21(1), 19-36.

11. Osmond, G.I., Lin, L., \& Seltzer, M.M. (2007). Mothers of adolescents and adults with autism: parenting multiple children with disabilities. Intellectual and Developmental Disabilities, 45(4), 257-270.

12. Piazza, C., \& Fisher, W. (2005). On the effectiveness of and preference for punishment and extinction components of function-based interventions. Journal of Applied Behavior Analysis, 38, 51-65.

13. Samadi, S.A., \&McConkey, R. (2011). Autism in developing countries: Lessons from Iran. Autism Research and Treatment, 2001(Article ID 145359), 1-11.

14. Schieve, L.A., Blumberg, S.J., Rice, C., Visser, S.N., \& Boyle, C. (2006). The relationship between autism and parenting stress. PediatricsS114DOI: 10.1542/peds.2006-2089Q Pediatrics 2007;119;\$114.

15. Sedrakyan, S.A. (2014). Family Psychology. Mania+studios LLC, Las Vegas, USA, p. 527

16. Shine, R., \& Perry, A. (2010). The relationship betweenparental stress and intervention outcome of children with autism. Journal on Developmental Disabilities, 16(2), 64-66.

17. Smith, L.E., Greenberg, J.S., Seltzer, M.M., \& Hong, J. (2008). Symptoms and behavior problems of adolescents and adults with autism: effects of mother-child relationship quality, warmth, and praise. American Journal on Mental Retardation, 113(5), 387-402.

18. Smith, L.E.,Hong, J., Seltzer, M.M., Greenberg, J.S., Almeida, D.M., \&Somer, L. (2010). Daily experiences among mothers of adolescents and adultswith autism spectrum disorder. Journal of Autism and Developmental Disorders, 40(2), 167-178. DOI 10.1007/s10803-009-0844-y.

19. Smith, L.E., Maenner, M.J., \& Seltzer, M.M. (2012). Developmental trajectories in adolescents and adults with autism:the case of daily living skills. Journal of the American Academy of Child \& Adolescent Psychiatry, 51(6), 622-631.

20. Sperry, L.A., \& Symons, F.J. (2003). Maternal judgments ofintentionality in young children with autism: the effects of diagnostic information and stereotyped behavior. Journalof Autism and Developmental Disorders, 33(3), 281-287.

21. Van Laarhoven, T., Kraus, E., Karpman, K., Nizzi, R., \& Valentino, J. (2010). A comparison of picture and video

22. Prompts to teach daily living skills to individuals with autism. Focus on Autism and Other Developmental Disabilities, 25(195), 195-208. 


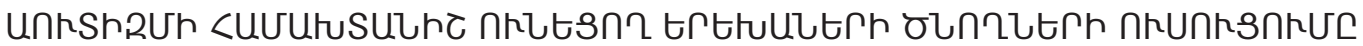

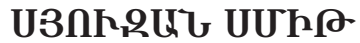

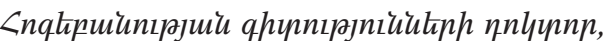

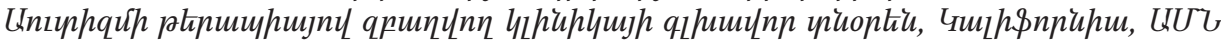

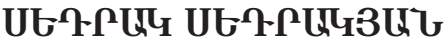

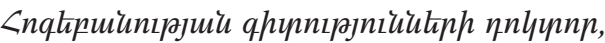

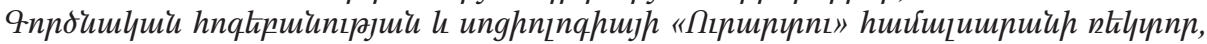

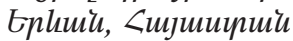

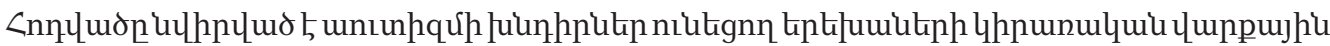

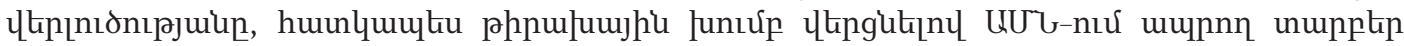

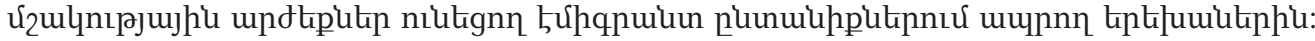

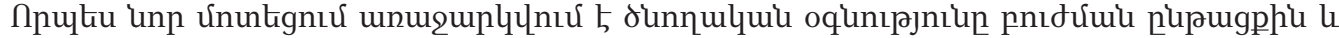

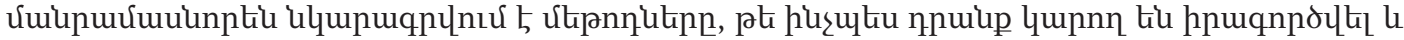

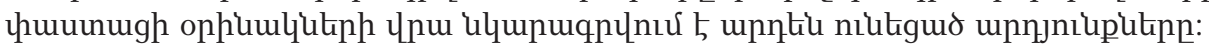

\section{ОБУЧЕНИЕ РОДИТЕЛЕЙ ДЕТЕЙ С ДИАГНОЗОМ “АУТИЗМ”}

СЮЗАН СМИТ

Доктор психологических наук

Главный клинический директор для интегративных методов леченияаутизма, Калифорния, США

\section{СЕДРАК СЕДРАКЯН}

Доктор психологических наук

Ректор университета практической психологии и социологии "Урарту”, Ереван, Армения

Статья посвящена проблемам детей с аутизмом в прикладной анализ поведения.Целевая группа принимается дети иммигрантов которые живут в США и каторые имеют разные культурные ценности.

Как новый подход предложен вмешательство родительей к методам лечения, и описаны в деталях, как они будут достигнуты. Так же приведены примеры результатов которые уже били достигнуты. 